\title{
Delay and distortion of slow light pulses by excitons in $\mathrm{ZnO}$
}

\author{
T. V. Shubina, ${ }^{1}$ M. M. Glazov, ${ }^{1}$ N. A. Gippius, ${ }^{2,3}$ A. A. Toropov, ${ }^{1}$ D. Lagarde,${ }^{2,4}$ P. Disseix,${ }^{2}$ J. Leymarie, ${ }^{2}$ \\ B. Gil, ${ }^{5}$ G. Pozina, ${ }^{6}$ J. P. Bergman, ${ }^{6}$ and B. Monemar ${ }^{6}$ \\ ${ }^{1}$ Ioffe Physical-Technical Institute, RAS, St. Petersburg 194021, Russia \\ ${ }^{2}$ LASMEA, UMR 6602, Université Blaise Pascal, F-63177 Aubiere Cedex, France \\ ${ }^{3}$ General Physics Institute RAS, Moscow 119991, Russia \\ ${ }^{4}$ LPCNO, UMR INSA-CNRS-UPS, Université de Toulouse, F-31077 Toulouse, France \\ ${ }^{5}$ L2C UMR CNRS 5221, Université Montpellier 2-CNRS, Montpellier, France \\ ${ }^{6}$ Department of Physics, Chemistry and Biology, Linköping University, S-58183 Linköping, Sweden
}

(Received 15 June 2011; published 1 August 2011)

\begin{abstract}
Distortion of light pulses in $\mathrm{ZnO}$ caused by both bound and free excitons is demonstrated by time-of-flight spectroscopy. Numerous lines of bound excitons dissect the pulse spectrum and induce slowdown of light propagation around the dips. Exciton-polariton resonances determine the overall pulse delay, which approaches $1.6 \mathrm{~ns}$ at $3.374 \mathrm{eV}$ for a $0.3 \mathrm{~mm}$ propagation length, as well as the pulse curvature in the time-energy plane and its attenuation. Analysis of $\mathrm{cw}$ and time-resolved data yields the excitonic parameters inherent for bulk $\mathrm{ZnO}$. A discrepancy is found between these bulk parameters and those given by surface-probing techniques.
\end{abstract}

DOI: 10.1103/PhysRevB.84.075202

PACS number(s): 42.50.Ct

\section{INTRODUCTION}

The phenomenon of light retardation in a dispersive medium has been studied from the beginning of the last century, ${ }^{1}$ for both local dielectric ${ }^{2}$ and exciton-polariton systems. ${ }^{3}$ Garrett and McCumber ${ }^{4}$ examined the propagation of a Gaussian light pulse near a resonant absorption line. In addition to exceptional variation of the group velocity, they predicted unchanged width and shape of the pulse for many exponential absorption depths. Early experiments showed no significant changes in the temporal shape of the pulse at the excitonic resonances in GaAs (Ref. 5) and GaP. ${ }^{6}$ The distortion of the pulse spectrum was not considered, while the optical dispersion implies a different group velocity and attenuation for the higher- and lower-energy edges of the pulse. ${ }^{7,8}$ That can result in pulse curvature in the time-energy plane and a shift of its maximum. The narrow absorption line can produce a hole in the pulse spectrum, causing temporal beats. ${ }^{9}$ More recent time-resolved (TR) measurements have found such features of pulse distortion in various semiconductors. ${ }^{10-14}$

Resumption of the interest in slow light was stimulated by possible applications in information processing. ${ }^{15}$ That requires a delay significantly exceeding the pulse duration, while their ratio is limited when quantum coherent effects are exploited. The resonant dispersion allows one, in principle, to realize a high ratio, but at the cost of severe signal attenuation. Wide-gap semiconductors, like $\mathrm{ZnO}$ and $\mathrm{GaN}$, possess strong excitonic resonances that makes them suitable for such light retardation. Light velocity as low as $6000 \mathrm{~km} / \mathrm{s}$ in ZnO (Ref. 13) and $2100 \mathrm{~km} / \mathrm{s}$ in GaN (Ref. 14) was measured below the bound exciton (BX) lines. In view of these findings, the question about the distortion of a light pulse has been revived. ${ }^{16}$

Here, we report on delay and distortion of light pulses by excitons in $\mathrm{ZnO}$. The results are interesting for two reasons: First, $\mathrm{ZnO}$ possesses numerous lines related to different states of donor-bound excitons. The fine spectrum of these excitons is probed by time-of-flight spectroscopy via the local distortion of the pulse shape at different energies. Second, simulation of the general shape of the transmitted pulse permits us to determine basic parameters of exciton-polariton resonances, inherent to bulk $\mathrm{ZnO}$. They turn out to be somewhat different from those given by surface-probing techniques.

\section{EXPERIMENTAL DETAILS}

The time-of-flight experiments were performed using freestanding $c$-plane $\mathrm{ZnO}$ samples with thickness $L=(0.3-2) \mathrm{mm}$ supplied by Tokyo Denpa Co. Pulses of a picosecond tunable laser (Mira-HP, second harmonic) hit the sample placed in a He cryostat. Their detected temporal width (15-30 ps) was determined by instrumental accuracy of the system as a whole (dependent on the jitter of the laser pulses and spectral resolution of the monochromator, etc.). A Hamamatsu streak camera with a 2 ps temporal resolution (for the fastest time range) was used to record the TR images of the collected light.

Two schemes of measurements were exploited. In the forward (transmission) geometry, excitation and detection are carried out from different sides of the sample. In the backward geometry, this is done from the same side [Fig. 1(a), inset]. In both cases, we were able to register a series of replicas, arising due to the light reflection from the crystal boundaries. Propagating like polaritons ${ }^{14}$ they cover a distance of $n L$, where $n$ is an odd (even) number for the forward (backward) geometry. Thus, their study is the same as the investigation of a set of samples with identical parameters but different lengths. The initial time moment when light impinges on the sample surface was determined in the forward geometry by means of measurements of the light pulse without the sample. In the backward geometry, we used for this purpose a light streak directly reflected from the surface ( $0 L$ in our notation), which precedes the series of replicas in a time-resolved image. This way is more precise because it excludes variation of the delay time due to readjustment between two different measurements.

Supplementary continuous-wave (cw) photoluminescence (PL), transmission, and reflection measurements were performed at different temperatures using a $\mathrm{He}-\mathrm{Cd}$ laser (for PL) 

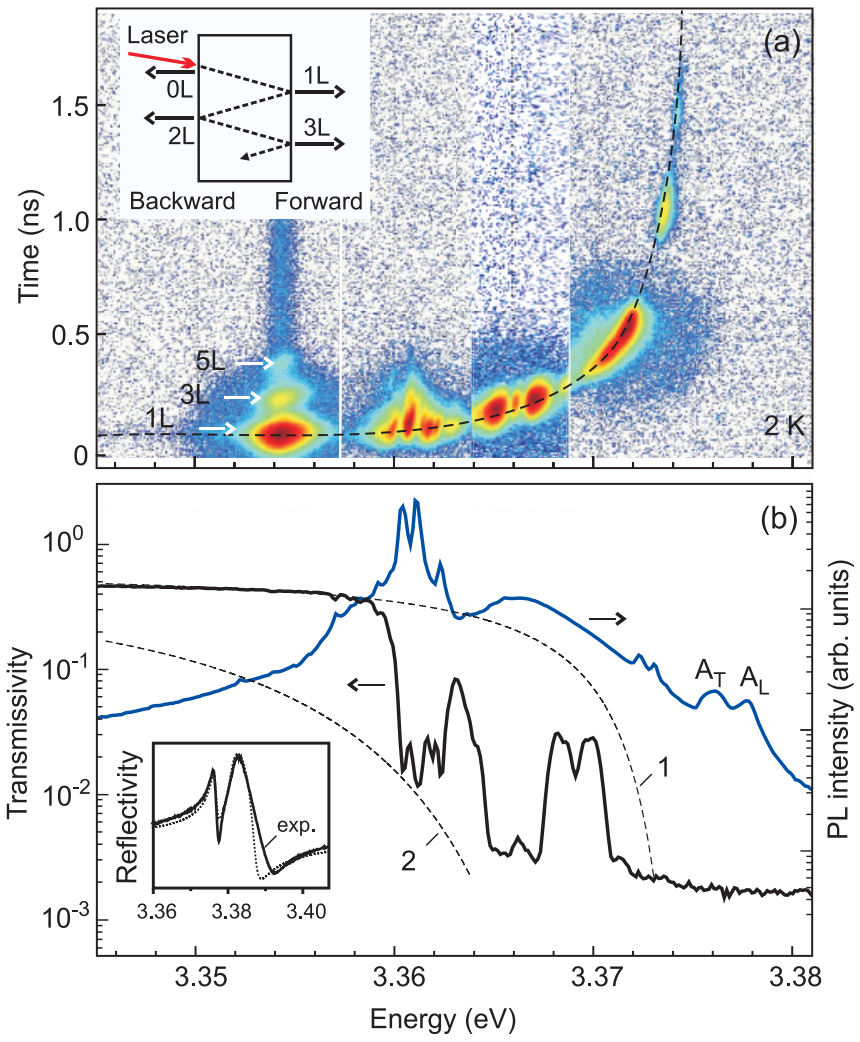

FIG. 1. (Color online) (a) Combined images of four pulses propagating through the $0.3 \mathrm{~mm}$ sample (logarithmic intensity scale). The boundaries between them are marked by white lines. The delay time dependence (dashed line) is calculated neglecting the BX lines to show the dominant contribution of the free-exciton resonances. The inset presents the scheme of the signal detections in the forward and backward geometries; labels $n L$ mark the light replicas. (b) Spectra of cw PL, transmission, and reflection (the inset) measured in the same sample. $A_{T}$ and $A_{L}$ denote the transverse and longitudinal $A$ exciton emission peaks. Dashed lines show the transmission spectra simulated neglecting the $\mathrm{BX}$ lines with $\hbar \Gamma_{j}$ (1) $3 \mu \mathrm{eV}$ and (2) $30 \mu \mathrm{eV}$.

or a tungsten lamp (for the others). In the PL spectra, the number (2-4) and intensity of the dominant BX lines vary among the samples, while their energies are almost identical.

\section{RESULTS AND DISCUSSION}

Typical images of the light pulses propagating at different energies are shown in Fig. 1(a). Below the BX lines, a series of replicas can be seen. The higher-energy edge of the curved light stripe is observed in the close vicinity of the $A$ exciton at $3.374 \mathrm{eV}$ with a delay of about $1.6 \mathrm{~ns}$. This light stripe gradually narrows due to increasing absorption. Further shift of the pulse toward higher energies results in its full quenching accompanied by an increase of the BX PL. These images apparently show that the general curvature and delay of the pulses follow the slowdown of the group velocity due to the optical dispersion controlled by the exciton-polariton resonances.

In contrast, the influence of the BX lines is spectrally local. They provide dips cutting the pulses into several parts and induce extra retardation of light nearby. Because even a very

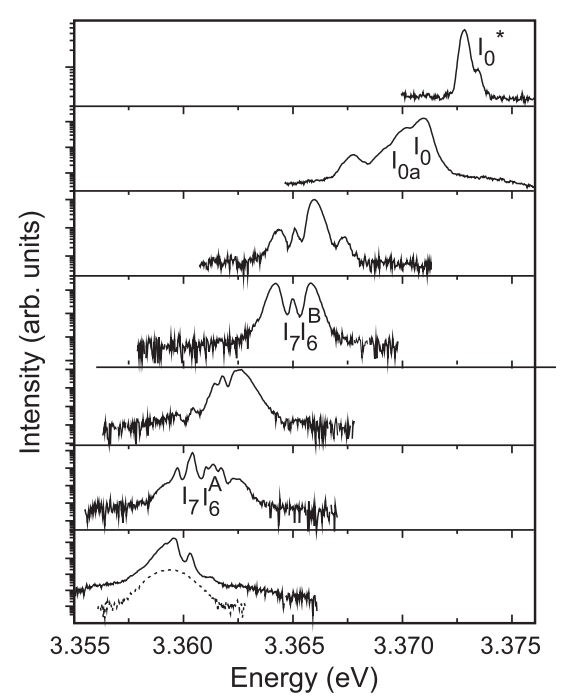

FIG. 2. Selected spectral cross sections of the light pulses passing the $0.3 \mathrm{~mm}$ sample, made at different delay times from $110 \mathrm{ps}$ (bottom) up to $1200 \mathrm{ps}$ (top). The dips corresponding to several important lines are labeled. The shape of the initial light pulse is shown by the dotted curve.

weak line causes noticeable absorption if a sample is thick enough, the number of BX lines resolved by the time-of-flight spectroscopy (similar to transmission) is higher than in the PL spectra [Fig. 1(b)]. The spectral cross sections of the pulses (Fig. 2) allow us to reconstruct the fine structure of the BX spectrum, which contains at least 18 lines in the 3.356-3.374 eV range (Table I). These lines can be divided into three groups: (i) Two sets of $A$ and $B$ excitons bound to neutral donors $D^{0} X$, separated by a gap of $\sim 4.5 \mathrm{meV}$. They produce the strongest local changes. (ii) Excitons bound to ionized donors $D^{+} X$, at higher energies. iii) Weak lines related to the vibrational-rotational (vr) states of the bound excitons. Besides, a few additional lines are discovered by this time-of-flight study.

To model the pulse delay and distortion, the spectrum of the impinging laser pulse $E_{0}(\omega)$ is approximated as a Gaussian with central frequency $\omega_{0}$ and the envelope maximum in the time domain at $t=0$. The amplitude and phase of the pulse in the frequency domain are given by the Fourier transform at the boundary of the medium, $z=0 .{ }^{8}$ In the linear regime, the

TABLE I. Parameters of bound excitons in bulk $\mathrm{ZnO}$ obtained through the pulse distortion analysis. The notation follows Ref. 19; $X$ denotes unidentified lines.

\begin{tabular}{lcclcc}
\hline \hline Transition & $E(\mathrm{eV})$ & $f_{\mathrm{BX}}$ & Transition & $E(\mathrm{eV})$ & $f_{\mathrm{BX}}$ \\
\hline$I_{0}^{*}\left(D^{+} X_{A}\right)$ & 3.3733 & $1 \times 10^{-7}$ & $X$ & 3.3635 & $5 \times 10^{-7}$ \\
$I_{0}\left(D^{+} X_{A}\right)$ & 3.3721 & $1 \times 10^{-6}$ & $I_{6}^{v r}\left(D^{0} X_{A}\right)$ & 3.3623 & $1 \times 10^{-6}$ \\
$I_{0 a}\left(D^{+} X_{A}\right)$ & 3.3717 & $1 \times 10^{-6}$ & $I_{7}^{v r}\left(D^{0} X_{A}\right)$ & 3.3620 & $1 \times 10^{-6}$ \\
$X$ & 3.3705 & $3 \times 10^{-7}$ & $I_{8}^{v r}\left(D^{0} X_{A}\right)$ & 3.3616 & $1 \times 10^{-6}$ \\
$X$ & 3.3695 & $3 \times 10^{-7}$ & $I_{5}\left(D^{0} X_{A}\right)$ & 3.3614 & $2 \times 10^{-6}$ \\
$X$ & 3.3685 & $1 \times 10^{-6}$ & $I_{6}\left(D^{0} X_{A}\right)$ & 3.3608 & $5 \times 10^{-6}$ \\
$I_{5}^{B}\left(D^{0} X_{B}\right)$ & 3.3669 & $1 \times 10^{-6}$ & $I_{7}\left(D^{0} X_{A}\right)$ & 3.3600 & $5 \times 10^{-6}$ \\
$I_{6}^{B}\left(D^{0} X_{B}\right)$ & 3.3653 & $2 \times 10^{-6}$ & $I_{8}\left(D^{0} X_{A}\right)$ & 3.3593 & $5 \times 10^{-9}$ \\
$I_{7}^{B}\left(D^{0} X_{B}\right)$ & 3.3647 & $2 \times 10^{-6}$ & $I_{9}\left(D^{0} X_{A}\right)$ & 3.3566 & $1 \times 10^{-9}$ \\
\hline \hline
\end{tabular}


electric field at the spatial point $z=L$ can be described as

$$
E(L, t)=\int_{-\infty}^{\infty} E_{0}(\omega) T(L, \omega) e^{-i \omega t} \frac{d \omega}{2 \pi} .
$$

Here, the amplitude transmission coefficient $T(L, \omega)$ takes into account the multiple reflections and attenuation of light impinging normally on a slab with parallel boundaries. ${ }^{17}$ It depends on the complex wave vector of light in the sample, $k(\omega)=(\omega / c) \sqrt{\varepsilon(\omega)}$, with $\varepsilon(\omega)$ being the dielectric function of the medium. The pulse shape in the time-frequency representation is calculated by means of the Gabor transform with the temporal window of $\sim 15$ ps. $^{18}$

For a medium with several exciton-polariton resonances, $\varepsilon(\omega)$ is written as $\varepsilon(\omega)=\varepsilon_{b}+\sum_{j} X_{j}$, where $\varepsilon_{b}$ is the background dielectric constant (taken as 8.1) and $X_{j}$ are the contributions of these resonances. When the resonances are assumed to be homogeneous, an expression taking into account the spatial dispersion can readily be used. ${ }^{20}$ With inhomogeneous broadening induced, e.g., by structural imperfections, each $X_{j}$ term can be represented by the convolution of the line with a Gaussian centered at the same frequency: ${ }^{14}$

$$
X_{j}=\int \frac{f_{j} \omega_{0, j}}{\omega_{0, j}+\beta k^{2}+\xi-i \Gamma_{j}-\omega} \frac{1}{\sqrt{\pi} \Delta_{j}} \exp \left(\frac{-\xi^{2}}{\Delta_{j}^{2}}\right) d \xi
$$

Here, each $j$ resonance is characterized by a frequency $\omega_{0, j}$, an oscillator strength $f_{j}$, and a damping term $\Gamma_{j} . \Delta_{j}$ describes the inhomogeneous width. Spatial dispersion is taken into account by the term $\beta k^{2}=\left(\hbar^{2} / 2 M_{j}\right) k^{2}$, where the effective masses $M_{j}$ are assumed to be infinite for the BX and equal to $0.9 m_{e}$ for each free-exciton resonance. Equations (1) and (2) account for the spatial dispersion in a simplified way. They are valid for $\omega$ not too close to $\omega_{0, j}$, which is sufficient for our purposes. Variation of the group velocity $v_{g}(\omega)=$ $d \omega / d k$, controlled mostly by $f_{j}$, determines the different delay $T=L / v_{g}$ of the pulse constituents and, hence, the pulse curvature.

In our simulations, all noticeable $\mathrm{BX}$ lines were taken into account. $\hbar \Gamma=1 \mu \mathrm{eV}$ and $\hbar \Delta=75 \mu \mathrm{eV}$ characterize these narrow lines. The $f_{\mathrm{BX}}$ values corresponding to the observed local distortion are given in Table I. For the sake of illustration, we present the modeling of the intricate pattern appearing when the central energy of the pulse falls into the gap between the closely situated $I_{6}$ and $I_{7}$ lines. The light propagates there as an extremely narrow $\sim 0.3 \mathrm{meV}$ stripe [Fig. 1(a)]. Figures 3(a)3(c) show that a twice higher $f$ value taken for the $D^{0} X_{B}$ transitions $\left(4 \times 10^{-6}\right.$ vs $\left.2 \times 10^{-6}\right)$ provides an unacceptably long delay and too strong distortion of the pulse.

To determine the parameters of the exciton-polariton resonances, we consider exclusively the data on the processes taking place inside the crystal, namely, cw transmission and pulse propagation. We abstain from using the data from reflection and PL spectroscopies, because they probe mostly a region close to the surface. The modeling is simplified owing to the fact that each excitonic parameter is responsible predominantly for some particular characteristics. The derived exciton-polariton parameters are collected in Table II (the distant $C$ exciton is neglected).

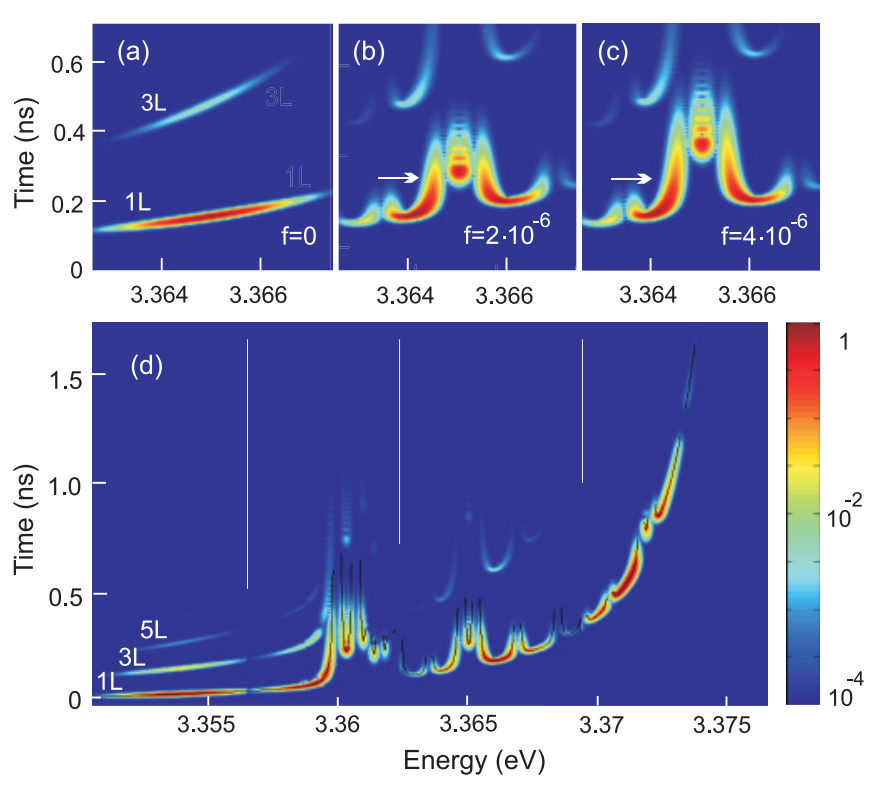

FIG. 3. (Color online) Simulations of the shapes of Gaussian light pulses propagating through the $0.3 \mathrm{~mm}$ sample. (a)-(c) A pulse centered at $3.365 \mathrm{eV}$ modeled (a) neglecting $\mathrm{BX}$ lines, and taking into account the BX lines with (b) $f=2 \times 10^{-6}$ and (c) $f=4 \times 10^{-6}$. Arrows mark the experimental delay of the pulse center. (d) Pulses centered at 3.354, 3.360, 3.366, and $3.373 \mathrm{eV}$ modeled using the data of Tables I and II. The black line presents the calculated delay dependency for relative intensity $>10^{-7}$. In each particular image with boundaries marked by white lines, the intensity (logarithmic scale) is normalized to the maximum value.

The modeling reveals two severe limitations:

(1) The damping term $\hbar \Gamma$ must be as small as $\sim 3 \mu \mathrm{eV}$ at low temperature. Higher values would result in full opaqueness in the transmission spectra in the range where the narrow BX lines are clearly resolved [Fig. 1(b)]. This is independent of the model used to describe $\varepsilon(\omega)$, because the absorption depends on the imaginary part of the dielectric function, which is determined by this term.

(2) The inhomogeneous width $\hbar \Delta$ cannot exceed $0.5 \mathrm{meV}$ for the $A$ exciton resonances. This restriction arises from the observation of the transmitted light at $3.374 \mathrm{eV}$. With stronger broadening, which may occur in crystals of worse quality or

TABLE II. Low-temperature exciton-polariton parameters derived from volume- and surface-probing measurements.

\begin{tabular}{lcc}
\hline \hline Parameter & A exciton & $B$ exciton \\
\hline \multicolumn{3}{c}{ TR and cw transmission } \\
$\hbar \omega_{0}(\mathrm{eV})$ & $3.376 \pm 0.0002$ & $3.382 \pm 0.001$ \\
$f$ & $0.0072 \pm 0.0002$ & $0.012 \pm 0.001$ \\
$\omega_{L T}(\mathrm{meV})$ & 3 & 5 \\
$\hbar \Gamma(\mu \mathrm{eV})$ & $3 \pm 0.2$ & $3.5 \pm 0.5$ \\
$\hbar \Delta(\mathrm{meV})$ & $<0.5$ & $<1$ \\
& PL and reflection & $3.382 \pm 0.001$ \\
$\hbar \omega_{0}(\mathrm{eV})$ & $3.3758 \pm 0.0002$ & 6.5 \\
$\omega_{L T}(\mathrm{meV})$ & $1.8 \pm 0.2$ & 1.5 \\
$\hbar \Gamma^{*}(\mathrm{meV})$ & 0.75 & \\
\hline \hline
\end{tabular}




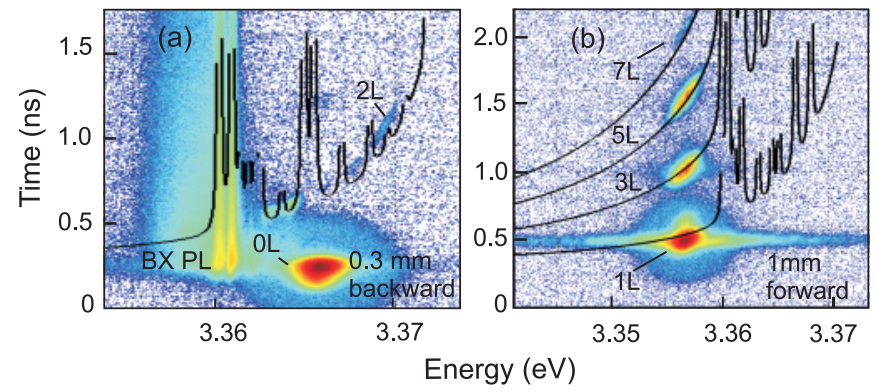

FIG. 4. (Color online) Images of $n L$ replicas recorded in the $0.3 \mathrm{~mm}$ (a) and $1 \mathrm{~mm}$ (b) samples in the backward and forward geometry, respectively. $0 L$ marks the pulse directly reflected from the surface. The black lines present the calculated delay dependencies shown for intensity of the transmitted light larger than $10^{-7}$ of the incident light intensity.

with a temperature rise, the pulse propagating at this energy would be fully attenuated.

These constraints based on the signal attenuation are more rigorous than a requirement imposed on the effective damping parameter, which should be less than the longitudinaltransverse splitting $\omega_{L T}$ to maintain the polaritonic modes. ${ }^{21}$ Moreover, our data do not exclude the possibility that the excitonic resonances inside the crystal are homogeneous with $\hbar \Gamma$ of a few $\mu \mathrm{eV}$.

As a result, we were able to simulate the TR images using the same exciton-polariton parameters for all samples (the BX parameters were slightly varied). Close agreement is observed between the simulated [Fig. 3(d)] and experimental [Fig. 1(a)] images of the pulses of different energies. Figure 4 presents the light replicas measured in the different samples. Their shapes are also well consistent with the calculated delay dependencies.
In addition, our modeling has revealed an obvious contradiction between the results of volume- and surface-probing experiments (Table II). The successful fitting of reflectivity spectra [Fig. 1(b), inset] requires the empirical damping term $\hbar \Gamma_{A}^{*}=0.75 \mathrm{meV}$ in the model of homogeneous resonances. ${ }^{20}$ A similar result can be obtained assuming the resonances as inhomogeneous with $\hbar \Delta_{A} \sim 0.9 \mathrm{meV}$. However, both variants would provide too strong attenuation of the propagating light. Further, the splitting between the PL peaks, ascribed to transverse and longitudinal $A$ exciton emission, is $1.6-2 \mathrm{meV}$, whereas the rough estimation using the bulk parameters gives $\omega_{L T} \approx 3 \mathrm{meV}$. This dependence on the measurement technique is suggestive of a certain deterioration of the excitonic resonances at the surface, which can be induced by modified structural properties. $^{22,23}$

\section{CONCLUSION}

In conclusion, a strong resonant delay of light pulses in $\mathrm{ZnO}$ is accompanied by their severe attenuation and shape distortion. Simulation of these effects enables us to determine the excitonic parameters of bulk $\mathrm{ZnO}$, as well as to establish that the resonances must have a low damping and limited broadening to allow the light propagation. Time-of-flight spectroscopy gives an opportunity to investigate the fine structure of a spectrum.

\section{ACKNOWLEDGMENTS}

This work has been supported in part by the RFBR, the Program of the Presidium of RAS, the Dynasty Foundation, and the FP7 ITN "Spin-Optronics" (237252). T.V.S. acknowledges the Université Montpellier 2 for its hospitality.
${ }^{1}$ For a review, see L. Brillouin, Wave Propagation and Group Velocity (Academic, New York, 1960).

${ }^{2}$ R. Loudon, J. Phys. A 3, 233 (1970).

${ }^{3}$ M. A. Bishop and A. A. Maradulin, Phys. Rev. B 14, 3384 (1976).

${ }^{4}$ C. G. B. Garrett and D. E. McCumber, Phys. Rev. A 1, 305 (1970).

${ }^{5}$ R. G. Ulbrich and G. W. Fehrenbach, Phys. Rev. Lett. 43, 963 (1979).

${ }^{6}$ S. Chu and S. Wong, Phys. Rev. Lett. 48, 738 (1982).

${ }^{7}$ M. D. Crisp, Phys. Rev. A 4, 2104 (1971).

${ }^{8}$ L. A. Vainshtein, Sov. Phys. Usp. 19, 189 (1976).

${ }^{9}$ M. D. Crisp, Phys. Rev. A 1, 1604 (1970).

${ }^{10}$ D. Fröhlich, A. Kulik, B. Uebbing, A. Mysyrowicz, V. Langer, H. Stolz, and W. von der Osten, Phys. Rev. Lett. 67, 2343 (1991).

${ }^{11}$ M. Kuwata, T. Kuga, H. Akiyama, T. Hirano, and M. Matsuoka, Phys. Rev. Lett. 61, 1226 (1988).

${ }^{12}$ T. Godde, I. A. Akimov, D. R. Yakovlev, H. Mariette, and M. Bayer, Phys. Rev. B 82, 115332 (2010).

${ }^{13}$ G. Xiong, J. Wilkinson, K. B. Ucer, and R. T. Williams, J. Phys.: Condens. Matter 17, 7287 (2005).
${ }^{14}$ T. V. Shubina, M. M. Glazov, A. A. Toropov, N. A. Gippius, A. Vasson, J. Leymarie, A. Kavokin, A. Usui, J. P. Bergman, G. Pozina, and B. Monemar, Phys. Rev. Lett. 100, 087402 (2008).

${ }^{15}$ M. Bigelow, N. Lepeshkin, and R. Boyd, Science 301, 200 (2003).

${ }^{16}$ D. S. Wiersma, Nature (London) 452, 942 (2008).

${ }^{17}$ L. D. Landau and E. M. Lifshitz, Electrodinamics of Continuous Media (Pergamon, Oxford, 1984).

${ }^{18}$ C. K. Chui, Wavelet Analysis and Its Applications Vol. 1: An Introduction to Wavelets (Academic Press, New York, 1992).

${ }^{19}$ B. K. Meyer, J. Sann, S. Eisermann, S. Lautenschlaeger, M. R. Wagner, M. Kaiser, G. Callsen, J. S. Reparaz, and A. Hoffmann, Phys. Rev. B 82, 115207 (2010).

${ }^{20}$ J. Lagois, Phys. Rev. B 16, 1699 (1977).

${ }^{21}$ M. Matsushita, J. Wicksted, and H. Z. Cummins, Phys. Rev. B 29, 3362 (1984).

${ }^{22}$ J. Lagois, Phys. Rev. B 23, 5511 (1981).

${ }^{23}$ B. Monemar, P. P. Paskov, J. P. Bergman, G. Pozina, A. A. Toropov, T. V. Shubina, T. Malinauskas, and A. Usui, Phys. Rev. B 82, 235202 (2010). 